\title{
Herpes zoster in psoriasis patients undergoing treatment with biological agents: prevalence, impact, and management challenges
}

This article was published in the following Dove Press journal:

Psoriasis: Targets and Therapy

18 October 2016

Number of times this article has been viewed

Lara el Hayderi

Fany Colson

Bita Dezfoulian

Arjen F Nikkels

Department of Dermatology, $\mathrm{CHU}$ du Sart Tilman, University Hospital of Liège, Liège, Belgium
Correspondence: Arjen F Nikkels Department of Dermatology, CHU du Sart Tilman, University Hospital of Liège, B-4000 Liège, Belgium

Tel +3243667232

$\mathrm{Fax}+3243667234$

Email af.nikkels@chu.ulg.ac.be

\begin{abstract}
As TNF- $\alpha$ is a major factor in the immune defense against herpes zoster (HZ); an increased incidence and severity of $\mathrm{HZ}$ cases were suspected in patients undergoing treatment with TNF antagonists. Several studies and clinical experience provided evidence that the incidence of $\mathrm{HZ}$ increases by twofold to threefold in this patient category. The number of severe cases of HZ, with multisegmental, disseminated cutaneous, and/or systemic involvement, is also increased. Concerning psoriasis patients under biologicals, the clinician should be more alert for an eventual $\mathrm{HZ}$ event, in particular during the first year of biological treatment, and be aware of the possibility of more severe $\mathrm{HZ}$ cases. HZ may also undergo an age-shift toward younger patients. Rapid identification of risk factors for severe HZ, such as severe prodromal pains and/ or the presence of satellite lesions, is recommended. The treatment recommendations of $\mathrm{HZ}$ in this patient group are identical to the recently published guidelines for the management of $\mathrm{HZ}$. The live attenuated viral vaccine OKA/Merck strain anti-HZ vaccination is recommended before initiating biological treatment in psoriasis patients. The new adjuvanted anti-HZ vaccine will probably also benefit patients while on biological treatment.
\end{abstract}

Keywords: herpes zoster, TNF antagonists, anti-IL17, anti-IL12/23, psoriasis, aciclovir

\section{Introduction}

Psoriasis is an inflammatory, predominantly skin disease, affecting $\sim 1 \%-5 \%$ of the population and has a high impact on the patient's quality of life. ${ }^{1}$ Moreover, severe and longstanding cases of psoriasis are often associated with a moderate-to-severe metabolic syndrome, potentially reducing the life expectancy by some years. ${ }^{1}$

Nowadays, moderate-to-severe plaque and patch-type psoriasis vulgaris can very effectively be treated with first-generation biologicals. They are represented by the TNF- $\alpha$-antagonists, ${ }^{2}$ including the receptor antagonist etanercept, a recombinant fusion protein that inhibits soluble and membrane-bound TNF- $\alpha^{3}$; the monoclonal chimeric antibody infliximab ${ }^{4}$ that binds membrane-bound and soluble TNF- $\alpha$; and the human monoclonal antibody adalimumab that blocks TNF- $\alpha$ interactions with the p55 and p75 cell surface TNF receptors. The TNF- $\alpha$-antagonists achieve PASI75 improvements in a high proportion of patients, ${ }^{5,6}$ a significant improvement compared to the older psoriasis treatments such as methotrexate, acitretin, and ciclosporin. ${ }^{2}$

Although the overall safety records of TNF- $\alpha$-antagonists are outstanding, there is evidence of an increased propensity to infections, ${ }^{7-10}$ in particular viral infections. Among these, varicella zoster virus (VZV), herpes simplex virus, hepatitis virus infections, and viral infections affecting the ear-nose-throat region are the most common. ${ }^{11-13}$ 
The second-generation biological agents for patch and plaque-type psoriasis include ustekinumab, which prevents the actions of IL-12 and IL-23 by binding to their mutual subunit $\mathrm{p} 40^{14}$; secukinumab, a human $\operatorname{IgG} 1 \kappa$ monoclonal antibody that selectively binds and neutralizes IL-17 $\mathrm{A}^{15,16}$; ixekizumab, a humanized IgG4 monoclonal antibody that neutralizes IL-17A ${ }^{17}$; and apremilast, a PDE4 inhibitor. ${ }^{18}$ The safety assessments of the initial trials demonstrated a similar rate of viral infections compared to the placebo groups. This fact may be due to the relative recent introduction of these agents in clinical practice. Another hypothesis is that the targeted cytokine pathways are less important for antiviral host defense mechanisms.

The cutaneous eruption-termed herpes zoster (HZ) is a self-limiting, dermatomally localized, papulo-vesicularpustular, and crusted eruption caused due to the reactivation of the VZV that remained dormant in the dorsal root ganglia after the primary contact with VZV in the form of chickenpox during childhood. ${ }^{19-21}$ Approximately 1 million new cases of $\mathrm{HZ}$ are diagnosed each year in the USA.22 Every year, 96 HZ-related deaths are reported, all diagnosed in elderly and/or immunocompromised patients. ${ }^{22}$ The incidence of $\mathrm{HZ}$ is rising with increasing age, and in patients older than 60 years, there are approximately ten cases of HZ per 1,000 US population per year. ${ }^{22}$ In patients aged between 35 years and 44 years, $\sim 194$ women and 261 men experience HZ per 100,000 population, with these values increasing to 1,624 women and 1,112 men in patients older than 75 years.

Severe and extensive cutaneous HZ, multidermatomal $\mathrm{HZ}$, and even systemic dissemination of VZV are the major complications of HZ. Especially, the immunocompromised population, including HIV patients, organ and bone marrow transplant recipients, and patients under immunosuppressive medication, is at risk for these complications. ${ }^{19-21}$

Postherpetic neuralgia (PHN) is the most feared complication following the resolution of the cutaneous lesions of HZ. The precise pathomechanisms are still not totally elucidated. The incidence of PHN also rises with increasing age. The risk of PHN is increased with, among the most important factors, age, severe cutaneous HZ, female sex, and/or severe prodromal pains. ${ }^{19-21}$ The term zoster-associated pain is used to describe the entire pain spectrum of $\mathrm{HZ}$, including the prodromal and concomitant pains as well as the PHN.

TNF- $\alpha$ is a major factor in the host immune response against HZ. ${ }^{23,24}$ Consequently, TNF- $\alpha$ antagonists could increase the risk and the severity of HZ. Indeed, since the earliest use of TNF- $\alpha$ antagonists, severe cases of HZ have continuously been reported. ${ }^{25}$ In contrast, for secukinumab, ixekizumab, and apremilast, not directly interfering with the TNF- $\alpha$ pathway, no reports have been published, until today, concerning severe $\mathrm{HZ}$ eruptions during treatment.

From the clinician's point of view, five major questions arise in relationship to the management of $\mathrm{HZ}$ in psoriasis patients under biological agents:

1. Is the incidence of $\mathrm{HZ}$ increased in psoriasis patients receiving biological agents?

2. Is the severity of $\mathrm{HZ}$ increased in psoriasis patients under biological agents?

3. Are the incidence and severity of PHN increased in psoriasis patients under biological agents?

4. Is anti-HZ vaccination indicated in patients before or while on biological treatment?

5. What are the recommendations in terms of management of $\mathrm{HZ}$ in patients with psoriasis receiving biologicals?

\section{Is the incidence of $\mathrm{HZ}$ increased in psoriasis patients receiving biological agents?}

This question is most frequently addressed by studying the crude incidence rates (CIRs) calculated by drug exposure and Cox proportional hazard models evaluating the adjusted association between a biological agent and the $\mathrm{HZ}$ event.

In general, these studies demonstrated that the exposition to biologicals increases the risk of $\mathrm{HZ}$ twofold to threefold, independent of whether these studies were dealing with inflammatory joint diseases, ${ }^{11,26-35}$ inflammatory intestinal bowel diseases, ${ }^{36,37}$ or inflammatory skin diseases. ${ }^{12,30,38-40}$ Whether there is a specific drug-associated risk remains still debated. An overview, including several cohort studies, randomized controlled trials, and case reports, suggested that infliximab conferred an increased risk of HZ, whereas adalimumab, etanercept, and ustekinumab did not. However, data still remain controversial. In sum, no specific biological agent seems to exhibit a particular $\mathrm{HZ}$ risk compared to another. ${ }^{12}$ Interestingly, the patients seem to be more at risk of developing $\mathrm{HZ}$ during the first months of treatment ${ }^{41}$ and the time to $\mathrm{HZ}$ seems to be shortened in patients treated with TNF- $\alpha$ antagonists. ${ }^{34}$

However, incidence data should be interpreted with caution for various reasons: the incidence of $\mathrm{HZ}$ increases progressively with age, a parameter often neglected in studies, and the study populations are extremely heterogeneous in many respects (previous exposure to one or more diseasemodifying antirheumatic drugs and duration of exposure, previous exposure to other biological agents and duration 
of exposure, other concomitant immunosuppression, other concomitant medication, combination therapies, etc).

Psoriasis is probably not different in terms of risk for $\mathrm{HZ}$ during biological treatment compared to the other inflammatory rheumatologic or gastrointestinal diseases. However, only a small number of studies specifically addressed this issue of psoriasis patients. ${ }^{12,38,39}$

An Israelian study evaluated the incidence of $\mathrm{HZ}$ among psoriasis patients treated with phototherapy, traditional systemic medications, and biological drugs. The incidence rates of $\mathrm{HZ}$ were calculated for each medication, and hazard ratios were adjusted for age, sex, and health care utilization burden. This study, which included a total of 22,330 psoriasis patients accounting for 215,656 patient years (py), revealed 1,321 cases of HZ. The CIRs were the following: 6.0 for ultraviolet B phototherapy (95\% confidence interval [CI]: 0-12.8), 10.1 for psoralen and ultraviolet A (95\% CI: 1.3-19), 5.4 for acitretin (95\% CI: 2.2-8.7), 17 for methotrexate (95\% CI: 10.6-23.4), 13.9 for etanercept (95\% CI: 0.3-27.4), 19.3 for infliximab (95\% CI: $0-45.8$ ), and 4.6 for controls (95\% CI: 4.3-5) per 1,000 py. No case of HZ was observed among patients treated with alefacept, efalizumab, or adalimumab. A multivariate analysis demonstrated that age, female sex, health care utilization pattern, and corticosteroid treatment were all associated with the time to HZ. Only the association of $\mathrm{HZ}$ with infliximab treatment approached statistical significance (hazard ratio: 1.77, 95\% CI: 0.92-3.43). ${ }^{38}$ The authors concluded that some biological drugs were associated with a higher incidence of $\mathrm{HZ}$ compared with controls, although not statistically significant. ${ }^{38}$ Another study included a total of 1,220 eligible patients on biologicals, representing 4,206 py. The CIR per 1,000 py was 5.2 (95\% CI: 3-7.4). Eleven HZ cases occurred during adalimumab treatment (CIR 7.1 per 1,000 py, 95\% CI: 2.9-11.3), four during etanercept treatment (CIR: 5.1 per 1,000 py, 95\% CI: 0.1-10), four during infliximab treatment (CIR: 2.4 per 1,000 py, 95\% CI: 0-4.7), two during ustekinumab treatment (CIR: 53.5 per 1,000 py, 95\% CI: 0-125.6), and one during rituximab treatment (CIR: 5.2 per 1,000 py, 95\% CI: 0-17.6). The incidence was higher for patients older than 60 years in this study, compared to that of the general population, although not statistically significant. Fourteen cases of HZ were observed in patients suffering from chronic inflammatory joint disease $(14 / 737=1.89 \%)$, five in those suffering from psoriasis $(5 / 238=2.1 \%)$, and three in those suffering from chronic inflammatory intestinal disease $(3 / 360=0.83 \%) .{ }^{39}$ Specifically assessing psoriasis patients, three cases of $\mathrm{HZ}$ were observed during etanercept treatment in 200 py, three cases of $\mathrm{HZ}$ during infliximab treatment in 166 py, zero cases of $\mathrm{HZ}$ during adalimumab treatment in 325 py, and two cases of $\mathrm{HZ}$ during ustekinumab treatment in 26 py. ${ }^{39}$ In total, five cases of $\mathrm{HZ}$ were observed during a total of 717 py $(0.7 \%)$ in psoriasis patients.

Consequently, considering that $\sim 1$ million patients will experience $\mathrm{HZ}$ each year in a total USA population of 320 million, ${ }^{22}$ representing $0.3125 \%$ of the population, it seems that biological treatments for psoriasis increase the risk of HZ by approximately twofold.

\section{Is the severity of $\mathrm{HZ}$ increased in psoriasis patients under biological agents?}

In the majority of psoriasis patients undergoing biological treatments, the course of $\mathrm{HZ}$ will not differ from $\mathrm{HZ}$ observed in a normal, nonimmunocompromised, age-matched population in terms of severity. However, the risk and incidence of severe $\mathrm{HZ}$ clearly increase in this population. Indeed, some of the patients may present very severe $\mathrm{HZ}$ in terms of cutaneous extension inside the involved dermatome(s), multidermatomal involvement in adjacent dermatomes and nonadjacent dermatomes, and increased duration of the $\mathrm{HZ}$ skin lesions (Figures 1 and 2). ${ }^{26-29,38,39,42,43}$ In a study that identified 86 cases of $\mathrm{HZ}$ among 82 patients under biologicals, multidermatomal $\mathrm{HZ}$ was observed in $18.3 \%$ of patients,

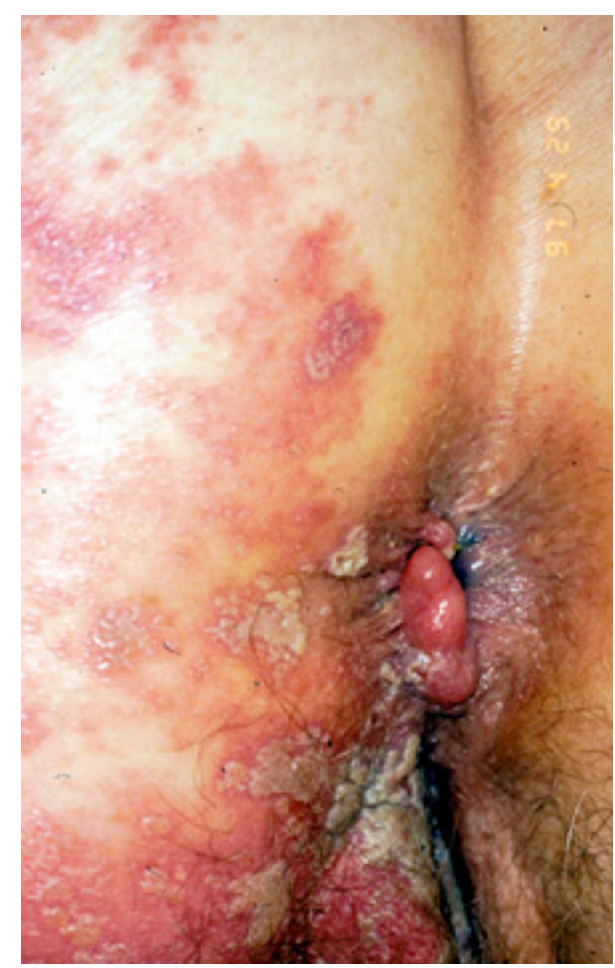

Figure I Severe and extensive multidermatomal unilateral $\mathrm{HZ}$ of the sacral dermatomes occurring during the use of TNF antagonists for psoriasis. Note: Photo courtesy of Professor Nikkels.

Abbreviation: $\mathrm{HZ}$, herpes zoster. 


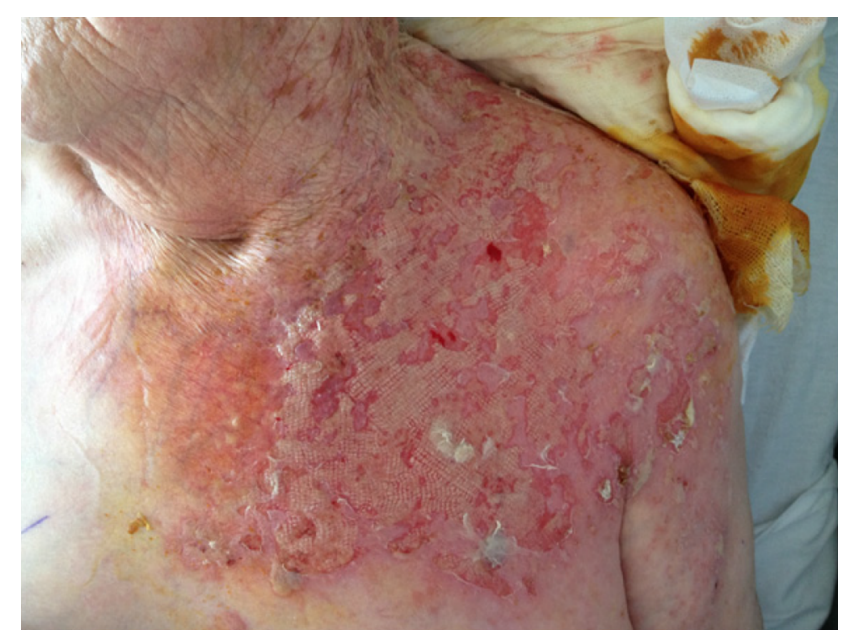

Figure 2 Severe multisegmental $\mathrm{HZ}$ in an elderly woman using TNF antagonists for psoriasis.

Note: Photo courtesy of Professor Nikkels.

Abbreviation: $\mathrm{HZ}$, herpes zoster.

HZ ophthalmicus in $4.9 \%$, and hospitalization due to severe disease in $14.6 \%$. Complications were reported in three patients. ${ }^{28}$ In another study, bidermatomal and multidermatomal $\mathrm{HZ}$ were observed in $45 \%$ and $32 \%$ of the $\mathrm{HZ}$ patients, respectively. Furthermore, two patients presented with a protracted course ( $>4$ weeks) of cutaneous VZV infection. ${ }^{39}$

It is not determined whether the cases of severe $\mathrm{HZ}$ were associated with a specific biologic agent. Currently, clinical experience cannot attribute a specific risk to any particular biological agent. Aside from potential severity, in this patient group, no distinctive clinical features of $\mathrm{HZ}$ were noted compared to common $\mathrm{HZ}$.

\section{Are the incidence and severity of PHN increased in psoriasis patients under biological agents?}

In the general population older than 50 years, the incidence of PHN is $\sim 2 \%-9 \%$ at 3 months after the resolution of the HZ skin lesions. ${ }^{19-21}$ Subsequently, the rate of PHN decreases progressively over months. TNF- $\alpha$ has been implicated in the pathogenesis of neuropathic pain. Higher circulating levels of $1 \beta$, IL-6, IL-8, IL-10, and TNF- $\alpha$ during HZ were measured compared to controls. Although IL-6 was significantly higher in $\mathrm{HZ}$ patients evolving to $\mathrm{PHN}$, there was no clear link between elevated TNF- $\alpha$ levels and PHN. ${ }^{44}$ The effect of biological treatments on the incidence and severity of PHN remains debated. One study that included 1,220 patients (4,206 py) receiving first-generation biologicals revealed that PHN, persisting for more than 6 months, in $20 \%$ of the $\mathrm{HZ}$ patients, ${ }^{39}$ was significantly higher compared to the general population. In contrast, in another patient group, only $2.4 \%$ of the patients under biologicals experienced PHN. ${ }^{28}$ Another study that included 206 patients presenting HZ while on TNF- $\alpha$ inhibitors only diagnosed PHN in $<1 \%$ of the patients. ${ }^{45}$

Hence, the precise impact of PHN on the first-generation biological treatments for psoriasis remains nonelucidated. No data are available for the second-generation biologicals.

\section{Is anti-HZ vaccination indicated in patients before or while on biological treatment?}

Owing to the increased incidence and risk of severe $\mathrm{HZ}$, vaccination against $\mathrm{HZ}$ seems a reasonable medical attitude. Vaccination against HZ aims to boost the VZV-specific cellmediated immunity. VZV-specific cell-mediated immunity is acquired during varicella and subsequently progressively wanes over the years until a threshold value is reached that allows VZV reactivation in the dorsal root ganglia, finally leading to $\mathrm{HZ}$.

The current $\mathrm{HZ}$ vaccine is a more concentrated ( 14-fold) form of the varicella vaccination OKA/Merck strain (Zostavax). This vaccine is a live attenuated viral vaccine (LAVV). Although highly efficacious with a $66.5 \%$ reduction in PHN $(P<0.001)$ and a $51.3 \%(P<0.001)$ reduction in the incidence of $\mathrm{HZ},{ }^{46}$ it is theoretically contraindicated to administer this type of vaccine during the use of TNF antagonists and other biological agents. However, in a retrospective cohort including 463,541 Medicare beneficiaries older than 60 years with rheumatoid arthritis, psoriasis, psoriatic arthritis, ankylosing spondylitis, or chronic inflammatory bowel diseases, the overall CIR of HZ was 7.8 cases per 1,000 py in the group having received the $\mathrm{HZ}$ vaccine and the rate among the unvaccinated was 11.6 cases per 1,000 py. Among the 633 patients exposed to biologicals at the time of vaccination, no single case of $\mathrm{HZ}$ occurred. ${ }^{47}$ Despite these reassuring results, it remains cautious to refrain from vaccinating with an LAVV system while on biologicals.

Recently, the efficacy and safety of a new adjuvanted $\mathrm{HZ}$ subunit vaccine containing the VZV glycoprotein E and the AS01B adjuvant system have been evaluated in a randomized, placebo-controlled, multicenter, Phase III study that included 15,411 adults older than 50 years of age and stratified according to age groups (50-59 years, 60-69 years, and older than 70 years). ${ }^{48}$ During a mean follow-up period of 3.2 years, $\mathrm{HZ}$ was confirmed in six participants among the 7,698 vaccinated volunteers (CI: 0.3 per 1,000 py) and in 210 participants 
among the 7,713 volunteers comprising the placebo group (CI: 9.1 per 1,000 py). The overall vaccine efficacy against HZ was 97.2\% (95\% CI: 93.7-99; $P<0.001)$. The efficacy of the vaccine in adults older than 70 years was similar to that observed in the younger age groups. ${ }^{48}$ The major advantage of this type of vaccine is that patients receiving biologicals are allowed to be vaccinated.

In sum, it is advisable to administer the LAVV-type anti$\mathrm{HZ}$ vaccine before initiating biologicals for psoriasis patients older than 50 years. ${ }^{49-52}$ For patients already under biologicals, the adjuvanted vaccine type will probably be recommended when commercially available. ${ }^{49}$

\section{What are the recommendations in terms of management of $\mathrm{HZ}$ in patients with psoriasis receiving biologicals?}

All the studies demonstrated that all the patients experiencing $\mathrm{HZ}$ responded positively to antiviral treatment, even the more severe cases. Resistant VZV strains were never encountered.

The treatment recommendations for $\mathrm{HZ}$ are not different from those for immunocompetent patients. ${ }^{49}$ Oral antiviral medication (acyclovir [ACV] $800 \mathrm{mg}$, five times per day for 7 days; valaciclovir $2 \times 500 \mathrm{mg}$, three times per day for 7 days; famciclovir $500 \mathrm{mg}$, three times per day for 7 days; or brivu$\operatorname{din} 125 \mathrm{mg} / \mathrm{d}$ for 7 days) is recommended for the subgroups of patients listed in Table 1. Intravenous administration of ACV (10 $\mathrm{mg} / \mathrm{kg} / 8 \mathrm{~h}$ for 7 days at least) is suggested for patients with complicated $\mathrm{HZ}$ or who are at a high risk of complicated HZ (Table 2). ${ }^{49}$ Antiviral treatment should be initiated as soon as possible ( $<72$ hours after the appearance of skin lesions). For ACV, famciclovir, and valaciclovir, the renal function should be tested before administration and dosages should be reduced in the case of renal insufficiency. The association of fluorouracil and brivudin should absolutely be avoided.

Table I Indications of oral antiviral medication

$\mathrm{HZ}$ of any localization in patients older than 50 years

Facial and/or cervical $\mathrm{HZ}$

$\mathrm{HZ}$ of any localization with

Moderate-to-severe ZAP

Hemorrhagic or necrotizing lesions

Multidermatomal involvement

Abnormal vesicles/presence of satellite lesions

Involvement of mucous membranes

$\mathrm{HZ}$ in immunocompromised patients

$\mathrm{HZ}$ in patients with predisposing skin diseases (such as atopic dermatitis)

Abbreviations: $\mathrm{HZ}$, herpes zoster; ZAP, zoster-associated pain.
Table 2 Indications of intravenous administration of ACV as suggested for patients with complicated $\mathrm{HZ}$ or who are at a high risk of complicated $\mathrm{HZ}$

$\mathrm{HZ}$ of the head and/or neck area, particularly in elderly patients $\mathrm{HZ}$ with hemorrhagic/necrotizing lesions, multisegmental involvement,

abnormal vesicles/satellite lesions, or involvement of mucous

membranes or generalized $\mathrm{HZ}$

$\mathrm{HZ}$ in immunocompromised patients

$\mathrm{HZ}$ with signs of visceral or central nervous system involvement

Abbreviations: $\mathrm{ACV}$, acyclovir; $\mathrm{HZ}$, herpes zoster.

No specific diagnostic techniques to prove the diagnosis of $\mathrm{HZ}$ are indicated for common $\mathrm{HZ}$ of the trunk. ${ }^{53}$ In contrast, $\mathrm{HZ}$ of the orofacial and anogenital area should be differentiated from zosteriform herpes simplex virus infections..$^{53}$

Although it is not possible to predict the severity of $\mathrm{HZ}$ in a given patient, the presence of satellite lesions at the beginning of the HZ eruption and the presence of a very painful prodrome are helpful clinical indicators for an increased risk of severe HZ. ${ }^{54}$

\section{Conclusion and recommendations}

Concerning $\mathrm{HZ}$ in psoriasis patients receiving biologicals, the following statements are proposed:

- Psoriasis does not confer a specific higher risk of HZ compared to the other inflammatory joint and intestinal diseases.

- The administration of biologicals in these patient groups increases the incidence of $\mathrm{HZ}$ by twofold to threefold.

- In patients treated with biologicals, one may expect HZ in younger age groups compared to common HZ.

- One should be more vigilant for a possible diagnosis of $\mathrm{HZ}$ in the first year after the instauration of a biological therapy.

- $\mathrm{HZ}$ is usually not different from common $\mathrm{HZ}$ in the immunocompetent population.

- Very severe and extensive cases of HZ are rare in these patient groups.

- In the case of HZ, look for risk factors for severe HZ, especially satellite lesions and severe prodromal pains.

- Antiviral treatment and management of $\mathrm{HZ}$ in psoriasis patients under biologicals are not different compared to their nonimmuncompromised counterparts.

- Intravenous ACV treatment is recommended for severe cases of $\mathrm{HZ}$.

- If possible, the HZ LAVV vaccine should be administered before starting biologicals in patients never having experienced HZ previously. 
- When commercially available, the new subunit HZ vaccine should be provided before or during biological treatments in patients never having experienced $\mathrm{HZ}$ previously.

\section{Disclosure}

The authors report no conflicts of interest in this work.

\section{References}

1. de la Brassinne M, Failla V, Nikkels AF. Psoriasis: state of the art 2013. Part I: clinical, historical, epidemiological and genetic aspects, co-morbidities and pathogenesis. Acta Clin Belg. 2013;68:427-432.

2. de la Brassinne M, Nikkels AF. Psoriasis: state of the art 2013. Part II: therapeutics. Acta Clin Belg. 2013;68:433-441.

3. Leonardi CL, Powers JL, Matheson RT, et al; Etanercept Psoriasis Study Group. Etanercept as monotherapy in patients with psoriasis. $N$ Engl J Med. 2003;349(21):2014-2022.

4. Reich K, Nestle FO, Papp K, et al; EXPRESS Study Investigators. Infliximab induction and maintenance therapy for moderate-tosevere psoriasis: a phase III, multicentre, double-blind trial. Lancet. 2005;366(9494):1367-1374.

5. Gordon KB, Langley RG, Leonardi C, et al. Clinical response to adalimumab treatment in patients with moderate to severe psoriasis: double-blind, randomized controlled trial and open-label extension study. J Am Acad Dermatol. 2006;55(4):598-606.

6. Kimball AB, Bensimon AG, Guerin A, et al. Efficacy and safety of adalimumab among patients with moderate to severe psoriasis with comorbidities: subanalysis of results from a randomized, double-blind, placebo-controlled, phase III trial. Am J Clin Dermatol. 2011;12(1):51-52.

7. Isaacs D. Infectious risks associated with biologics. Adv Exp Med Biol. 2013;764:151-158.

8. Pereira R, Lago P, Faria R, Torres T. Safety of anti-TNF therapies in immune-mediated inflammatory diseases: focus on infections and malignancy. Drug Dev Res. 2015;76(8):419-427.

9. Murdaca G, Spanò F, Contatore M, et al. Infection risk associated with anti-TNF- $\alpha$ agents: a review. Expert Opin Drug Saf. 2015;14(4):571-582.

10. Goh L, Jewell T, Laversuch C, Samanta A. A systematic review of the influence of anti-TNF on infection rates in patients with rheumatoid arthritis. Rev Bras Reumatol. 2013;53:501-515.

11. Che H, Lukas C, Morel J, Combe B. Risk of herpes/herpes zoster during anti-tumor necrosis factor therapy in patients with rheumatoid arthritis. Systematic review and meta-analysis. Joint Bone Spine. 2014;81(3):215-221.

12. Adelzadeh L, Jourabchi N, Wu JJ. The risk of herpes zoster during biological therapy for psoriasis and other inflammatory conditions. $J$ Eur Acad Dermatol Venereol. 2014;28(7):846-852.

13. Cacciapaglia F, Zuccaro C, Iannone F. Varicella-zoster virus infection in rheumatoid arthritis patients in the anti-tumour necrosis factor era. Clin Exp Rheumatol. 2015;33(6):917-923.

14. Griffiths CEM, Strober BE, van de Kerkhof P, et al; ACCEPT Study Group. Comparison of ustekinumab and etanercept for moderate-tosevere psoriasis. $N$ Engl J Med. 2010;362(2):118-128.

15. Langley RG, Elewski BE, Lebwohl M, et al; ERASURE Study Group; FIXTURE Study Group. Secukinumab in plaque psoriasis - results of two phase 3 trials. N Engl J Med. 2014;71(4):326-338.

16. Thaçi D, Blauvelt A, Reich K, et al. Secukinumab is superior to ustekinumab in clearing skin of subjects with moderate to severe plaque psoriasis: CLEAR, a randomized controlled trial. JAm Acad Dermatol. 2015;73(3):400-409.

17. Gordon KB, Blauvelt A, Papp KA, et al; UNCOVER-1 Study Group; UNCOVER-2 Study Group; UNCOVER-3 Study Group. Phase 3 trials of ixekizumab in moderate-to-severe plaque psoriasis. $N$ Engl J Med. 2016;375(4):345-356.
18. Bissonnette R, Pariser DM, Wasel NR, et al. Apremilast, an oral phosphodiesterase-4 inhibitor, in the treatment of palmoplantar psoriasis: results of a pooled analysis from phase II PSOR-005 and phase III efficacy and safety trial evaluating the effects of apremilast in psoriasis (ESTEEM) clinical trials in patients with moderate to severe psoriasis. J Am Acad Dermatol. 2016;75(1):99-105.

19. Arvin AM. Varicella-zoster virus. Clin Microbiol Rev. 1996;9(3):361-381.

20. Gershon AA, Gershon MD. Pathogenesis and current approaches to control of varicella-zoster virus infections. Clin Microbiol Rev. 2013;26(4):728-743.

21. Gilden D, Nagel MA, Cohrs RJ. Varicella-zoster. Handb Clin Neurol. 2014;123:265-283.

22. CDC [webpage on the Internet]. Shingles (Herpes Zoster). Available from: www.cdc.gov/shingles. Accessed August 23, 2016.

23. Nikkels AF, Sadzot-Delvaux C, Piérard GE. Absence of ICAM-1 expression in varicella zoster virus infected keratinocytes during herpes zoster. Another immune evasion strategy? Am J Dermatopathol. 2004;26:27-32.

24. Arvin AM, Moffat JF, Sommer M, et al. Varicella-zoster virus T cell tropism and the pathogenesis of skin infection. Curr Top Microbiol Immunol. 2010;342:189-209.

25. Tresch S, Trueb RM, Kamarachev J, French LE, Hofbauer GF. Disseminated herpes zoster mimicking rheumatoid vasculitis in a rheumatoid arthritis patient on etanercept. Dermatology. 2009;219(4):347-349.

26. Wendling D, Streit G, Toussirot E, Prati C. Herpes zoster in patients taking TNFalpha antagonists for chronic inflammatory joint disease. Joint Bone Spine. 2008;75(5):540-543.

27. McDonald JR, Zeringue AL, Caplan L, et al. Herpes zoster risk factors in a national cohort of veterans with rheumatoid arthritis. Clin Infect Dis. 2009;48(10):1364-1371.

28. Strangfeld A, Listing J, Herzer P, et al. Risk of herpes zoster in patients with rheumatoid arthritis treated with anti-TNF-alpha agents. JAMA. 2009;301(7):737-744.

29. García-Doval I, Pérez-Zafrilla B, Descalzo MA, et al; BIOBADASER 2.0 Study Group. Incidence and risk of hospitalisation due to shingles and chickenpox in patients with rheumatic diseases treated with TNF antagonists. Ann Rheum Dis. 2010;69(10):1751-1755.

30. Serac G, Tubach F, Mariette X, et al. Risk of herpes zoster in patients receiving anti-TNF- $\alpha$ in the prospective French RATIO registry. $J$ Invest Dermatol. 2012;132:726-729.

31. Curtis JR, Xie F, Yun H, Bernatsky S, Winthrop KL. Real-world comparative risks of herpes virus infections in tofacitinib and biologictreated patients with rheumatoid arthritis. Ann Rheum Dis. Epub 2016 Apr 25.

32. Yun H, Xie F, Delzell E, et al. Risks of herpes zoster in patients with rheumatoid arthritis according to biologic disease-modifying therapy. Arthritis Care Res (Hoboken). 2015;67(5):731-736.

33. Zisman D, Bitterman H, Shalom G, et al. Psoriatic arthritis treatment and the risk of herpes zoster. Ann Rheum Dis. 2016;75(1):131-135.

34. Segan J, Staples MP, March L, Lassere M, Chakravarty EF, Buchbinder R. Risk factors for herpes zoster in rheumatoid arthritis patients: the role of tumour necrosis factor- $\alpha$ inhibitors. Intern Med J. 2015;45(3):310-318.

35. Winthrop KL, Baddley JW, Chen L, et al. Association between the initiation of anti-tumor necrosis factor therapy and the risk of herpes zoster. JAMA. 2013;309(9):887-895.

36. Long MD, Martin C, Sandler RS, Kappelman MD. Increased risk of herpes zoster among 108604 patients with inflammatory bowel disease. Aliment Pharmacol Ther. 2013;37(4):420-429.

37. Ford AC, Peyrin-Biroulet L. Opportunistic infections with antitumor necrosis factor- $\alpha$ therapy in inflammatory bowel disease: meta-analysis of randomized controlled trials. Am J Gastroenterol. 2013;10(8):1268-1276.

38. Dreiher J, Kresch FS, Comaneshter D, Cohen AD. Risk of herpes zoster in patients with psoriasis treated with biologics. J Eur Acad Dermatol Venereol. 2012;26:1127-1132. 
39. Failla V, Jacques J, Castronovo C, Nikkels AF. Herpes zoster in patients treated with biologicals. Dermatology. 2012;224(3):251-256.

40. Di Costanzo L, Ayala F, Megna M, Gaudiello F, Patrì A, Balato N. The risk of herpes zoster in the anti-TNF- $\alpha$ era: a case report and review of the literature. J Dermatol Case Rep. 2013;7(1):1-4.

41. Strangfeld A, Eveslage M, Schneider M, et al. Treatment benefit or survival of the fittest: what drives the time-dependent decrease in serious infection rates under TNF inhibition and what does this imply for the individual patient? Ann Rheum Dis. 2011;70(11):1914-1920.

42. Failla V, Nikkels AF. Ustekinumab and herpes zoster. Dermatology. 2011;222(2):119-122.

43. Failla V, Castronovo C, Meex C, Nikkels AF. Protracted herpes zoster and severe postherpetic neuralgia after inadvertant infliximab administration. Eur J Dermatol. 2011;21:782-783.

44. Zhu SM, Liu YM, An ED, Chen QL. Influence of systemic immune and cytokine responses during the acute phase of zoster on the development of postherpetic neuralgia. J Zhejiang Univ Sci B. 2009;10(8): 625-630.

45. Javed S, Kamili QU, Mendoza N, Tyring SK. Possible association of lower rate of postherpetic neuralgia in patients on anti-tumor necrosis factor- $\alpha$. J Med Virol. 2011;83(11):2051-2055.

46. Oxman MN, Levin MJ, Johnson GR, et al; Shingles Prevention Study Group. A vaccine to prevent herpes zoster and postherpetic neuralgia in older adults. $N$ Engl J Med. 2005;352(22):2271-2284.
47. Zhang J, Xie F, Delzell E, et al. Association between vaccination for herpes zoster and risk of herpes zoster infection among older patients with selected immune-mediated diseases. JAMA. 2012;308(1):43-49.

48. Lal H, Cunningham AL, Godeaux O, et al; ZOE-50 Study. Efficacy of an adjuvanted herpes zoster subunit vaccine in older adults. $N$ Engl $J$ Med. 2015;372(22):2087-2096.

49. Werner RN, Nikkels AF, Marinovic B, et al. European consensus-based guideline (S2K) on the management of herpes zoster. Part 2: treatment. $J$ Eur Acad Dermatol Venereol. In press.

50. Nordgaard-Lassen I, Dahlerup JF, Belard E, et al; Danish Society for Gastroenterology. Guidelines for screening, prophylaxis and critical information prior to initiating anti-TNF-alpha treatment. Dan Med J. 2012;59(7):C4480.

51. Tran CT, Ducancelle A, Masson C, Lunel-Fabiani F. Herpes zoster: risk and prevention during immunomodulating therapy. Joint Bone Spine. Epub 2016 May 27.

52. Nisar MK, Ostor AJ. TNF antagonists and shingles: is vaccination advisable? Ann Rheum Dis. 2013;72:e1.

53. Werner RN, Nikkels AF, Marinovic B, et al. European consensus-based guideline (S2K) on the management of herpes zoster. Part 1: diagnostic means. J Eur Acad Dermatol Venereol. In press.

54. El Hayderi L, Bontems S, Nikkels-Tassoudji N, et al. Satellite lesions accompanying herpes zoster: a new prognostic sign for high-risk zoster Br J Dermatol. 2015;172(6):1530-1534.
Psoriasis: Targets and Therapy

\section{Publish your work in this journal}

Psoriasis: Targets and Therapy is international, peer-reviewed, open access journal focusing on psoriasis, nail psoriasis, psoriatic arthritis and related conditions, identification of therapeutic targets and the optimal use of integrated treatment interventions to achieve improved outcomes

\section{Dovepress}

and quality of life. Visit http://www.dovepress.com/testimonials.php to read real quotes from published authors. 